\title{
Activity-dependent Enhancement of Presynaptic Facilitation Provides a Cellular Mechanism for the Temporal Specificity of Classical Conditioning in Aplysia
}

Gregory A. Clark, ${ }^{1,4}$ Robert D. Hawkins, ${ }^{2}$ and Eric R. Kandel ${ }^{3}$

${ }^{1}$ Program in Neuroscience, Psychology Department

Princeton University

Princeton, New Jersey 08544

${ }^{2}$ Center for Neurobiology and Behavior

College of Physicians and Surgeons of Columbia University and New York State Psychiatric Institute

New York, New York 10032

${ }^{3}$ Howard Hughes Medical Institute

Center for Neurobiology and Behavior

College of Physicians and Surgeons of Columbia University and New York State Psychiatric Institute

New York, New York 10032

\section{Abstract}

A hallmark of many forms of classical conditioning is a precise temporal specificity: Learning is optimal when the conditioned stimulus (CS) slightly precedes the unconditioned stimulus (US), but the learning is degraded at longer or backward intervals, consistent with the notion that conditioning involves learning about predictive relationships in the environment. To further examine the cellular mechanisms contributing to the temporal specificity of classical conditioning of the siphon-withdrawal response in Aplysia, we paired action potential activity in siphon sensory neurons (the neural CS) with tail nerve shock (the US) at three critical time points. We found that CS-US pairings at short $(0.5 \mathrm{sec})$ forward intervals produced greater synaptic facilitation at sensorimotor connections than did either 0.5 -sec backward pairings or longer $(5 \mathrm{sec})$ forward pairings, as reflected in a differential increase in both the amplitude and rate of rise of the synaptic potential. In the same preparations, forward pairings also

${ }^{4}$ Corresponding author. differentially reduced the sensory neuron afterhyperpolarization relative to backward pairings, suggesting that changes in synaptic efficacy were accompanied by temporally specific changes in ionic currents in the sensory neurons. Additional experiments demonstrated that short forward pairings of sensory cell activity and restricted applications of the neuromodulatory transmitter serotonin (normally released by the US) differentially enhanced action potential broadening in siphon sensory neurons, relative to backward pairings. Taken together, these results suggest that temporally specific synaptic enhancement engages both spike-width-dependent and spike-width-independent facilitatory processes and that activity-dependent enhancement of presynaptic facilitation may contribute to both the CS-US sequence and proximity requirements of conditioning.

\section{Introduction}

Several forms of associative learning in mammals exhibit a precise temporal specificity (Hearst 1988; Rescorla 1988). A similar temporal specificity is evident in classical conditioning of the si-

LEARNING \& MEMORY 1:243-257 (c) 1994 by Cold Spring Harbor Laboratory Press ISSN1072-0502/94 \$5.00

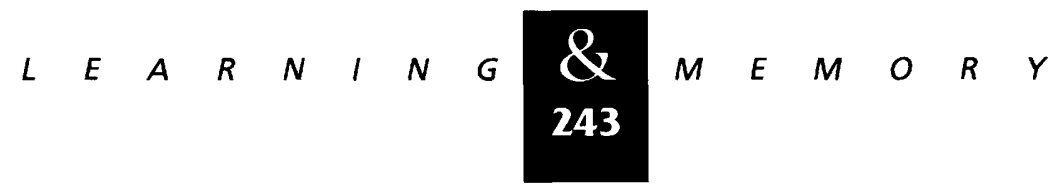


phon-withdrawal response in the marine mollusc Aplysia californica (Carew et al. 1981, 1983; Hawkins et al. 1986). Learning is optimal when the tactile siphon conditioned stimulus (CS) precedes the tail shock unconditioned stimulus (US) by 500 msec; longer CS-US intervals, backward pairings (in which the CS follows the US), and explicitly unpaired presentations of the CS and US all result in relatively less enhancement of the siphon-withdrawal response. Thus, conditioning is strongest when the CS precedes the US by a brief time interval and declines when this interval is either too long or too short. The nonmonotonic shape of the interstimulus interval learning function in Aplysia is a nearly universal feature of conditioning and is found in a wide variety of other species and tasks, although the exact values for effective conditioning vary with the particular paradigm and the nature and time course of the conditioned response (Hearst 1988; Rescorla 1988). This generality suggests the possibility of shared mechanisms.

The strong dependence of associative learning on the interstimulus interval (ISI) has important implications for the cognitive and, ultimately, biological processes underlying associative learning (Hearst 1988; Rescorla 1988). For example, although the temporal proximity, or contiguity, of the CS and the US can be equivalent in forward and backward pairing, the predictive value of the CS in the two paradigms is fundamentally different: In forward pairing, the CS precedes and predicts the occurrence of the US, whereas in backward pairing, the CS follows the US and thus predicts a period of US absence, because the US has already occurred (Moscovitch and LoLordo 1968). Similarly, at short CS-US intervals, the CS localizes the US more accurately in time than at long intervals. The greater associative learning resulting with short forward conditioning therefore supports the notion that classical conditioning can serve as a prototype for learning about predictive relationships in the environment.

What then are the physiological substrates that mediate the temporal specificity of classical conditioning? Previous experiments in Aplysia have indicated that a cellular mechanism termed activity-dependent enhancement of presynaptic facilitation (Hawkins et al. 1983; Carew et al. 1984) or activity-dependent neuromodulation (Walters and Byrne 1983a) contributes to the pairing specificity of classical conditioning of the siphon-withdrawal response (i.e., the difference between the effects of paired and unpaired training). Presentations of the US by itself activate a group of facilitatory interneurons that project to and produce presynaptic facilitation at connections of the LE siphon sensory cells (Hawkins et al. 1981b; Kandel and Schwartz 1982; Hawkins and Schacher 1989; Mackey et al. 1989). But when spike activity in siphon sensory neurons is paired with US presentation, as occurs during classical conditioning, this activity amplifies the normal facilitatory effects of the US. Thus, this pairing results in greater synaptic facilitation at sensory cell connections than does presentation of the US alone, or explicitly unpaired stimulation in which the CS and US are presented at widely spaced intervals of time (Hawkins et al. 1983; Walters and Byrne 1983a; Carew et al. 1984; Eliot et al. 1994). We now wished to test directly whether this mechanism might encode the temporal specificity of conditioning as well and, in particular, whether it is sensitive to both the proximity and the order of the CS and US. Thus, we asked two questions: First, can activity-dependent enhancement discriminate between 0.5-sec forward and 0.5-sec backward CS-US pairings? Second, can activity-dependent enhancement discriminate between CS-US pairings at short forward intervals $(0.5 \mathrm{sec})$ and pairings at slightly longer forward intervals ( $5 \mathrm{sec}$ )? Our findings indicate that activity-dependent enhancement of presynaptic facilitation exhibits a precise temporal specificity and could thus contribute to the temporal specificity of conditioning evident on the behavioral level.

\section{Materials and Methods}

Adult Aplysia (100-150 grams) were anesthetized with injection of isotonic $\mathrm{MgCl}_{2}$ (approximately half of body weight). In experiments examining synaptic facilitation, the abdominal ganglion (containing the siphon sensory and motor neurons), the pleural-pedal connectives, and the circumesophageal ganglia along with the attached tail (pedal) nerves were then removed, and the connective tissue encapsulating the abdominal ganglion was lightly fixed with glutaraldehyde $(0.5 \%, 30 \mathrm{sec})$ to mechanically stabilize the ganglion. The preparation was pinned to the Sylgard (Dow-Corning) floor of a chamber containing an equal mixture of isotonic $\mathrm{MgCl}_{2}$ and artificial seawater. The abdominal ganglion was partially desheathed to facilitate intracellular recording,

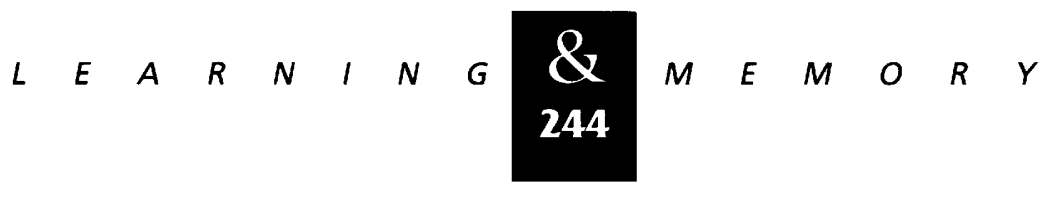


and the two tail nerves were placed either in suction electrodes or around chlorided silver posts for extracellular stimulation. During subsequent electrophysiological experiments, preparations were perfused with room temperature artificial seawater with the following composition (in $\mathrm{mM}$ ): $\mathrm{NaCl}, 460 ; \mathrm{KCl}, 10 ; \mathrm{CaCl}_{2}, 11 ; \mathrm{MgCl}_{2}$, 55; HEPES$\mathrm{NaOH}, 10$; plus amino acids, vitamins and sugars; pH 7.6.

We first examined synaptic plasticity induced by neural analogs of behavioral training at connections of siphon sensory cells. In separate experiments, we compared the effects of a 0.5 -sec forward pairing condition with either a 0.5 -sec backward pairing condition or a 5 -sec forward pairing condition. In brief, we recorded intracellularly from two LE siphon sensory neurons and a siphon motor neuron or interneuron that received a direct input from both sensory neurons. The majority of postsynaptic targets were probably $L_{\mathrm{S}} \mathrm{si}$ phon motor neurons, as defined by electrophysiological criteria (Frost et al. 1988). We then tested the monosynaptic excitatory postsynaptic potential (PSP) elicited in the postsynaptic cell by each sensory neuron before and after training, by stimulating each sensory neuron intracellularly to evoke an action potential (Fig. 1A, below). We also measured the amplitude of the afterhyperpolarization (AHP) that followed the test spike in the sensory neuron and the sensory neuron membrane potential during training trials.

The testing and training protocol utilized an AB design. Each of the two series consisted of four pretraining test trials, two paired training trials, and two post-training test trials, with an intertrial interval of $3 \mathrm{~min}$ throughout. During test trials, we elicited a single action potential separately in each of the two sensory neurons via intracellular stimulation to test the EPSP evoked in the postsynaptic target. Intracellular command pulses were $>10$ msec throughout the experiment to ensure reliable activation of the sensory neurons, particularly during the training trial spike trains, during which sensory neurons undergo accommodation. We also measured the input resistance of the postsynaptic cell on test trials via a hyperpolarizing command pulse. During the training trials, we paired spike train activity $(10 \mathrm{~Hz}$, seven spikes) in sensory neurons (the neural analog of the CS) with tail nerve shock US. This CS activity is comparable to that evoked by a tactile siphon CS during behavioral conditioning in semi-intact preparations (T. Cohen, V. Henzi, L. Frost, E.R. Kandel, and R.D.
Hawkins, unpubl.; see also Byrne et al. 1978). The CS spike train was presented at a different ISI for each of the two sensory neurons. For each preparation, one sensory neuron received 0.5 -sec forward pairing (CS onset preceded US onset by 0.5 sec), whereas the other sensory neuron received either 0.5-sec backward pairing or 5-sec forward pairing. Because CS stimulation was delivered directly to the sensory cell, bypassing normal conduction time down the siphon nerve, we also defined US onset relative to physiological activation, as reflected in time to onset of tail-shock-evoked PSPs in siphon motor neurons. The initial pairing conditions for the two sensory cells were determined by coin flip after the last pretraining test. To control for possible intrinsic differences in facilitation between the two cells, we rested the preparation for $15 \mathrm{~min}$ and then conducted a second training series in which the pairing conditions of the two cells was reversed; thus, each cell was tested under both ISI conditions. In the experiments comparing 0.5 -sec forward pairing and 0.5 sec backward pairing, the tail nerve shock US ( 1 sec, $10-$ to $20-\mathrm{Hz}$ train; 5 -msec pulses) was delivered via suction electrodes; US intensity (mean $=3.5 \mathrm{~V}$ ) was set independently for each tail nerve to 1.2-2 times the threshold for a single stimulus to elicit an excitatory PSP in the siphon motor neuron. Experiments comparing the effects of 0.5 -sec forward pairing and 5 -sec forward pairing used the same basic design. However, in these experiments, the tail nerve shock US ( 1.5 -sec train, $20 \mathrm{~Hz}$ ) was delivered via chlorided silver posts rather than suction electrodes, so US intensity was set at higher values $(100 \mathrm{~V}, 3-10$ times threshold) to overcome the partial shunting of current by seawater.

In additional experiments, we investigated the effects of forward and backward pairing on changes in sensory neuron action potential duration, which are believed to contribute to changes in synaptic strength at sensory cell connections. These experiments were performed with the isolated abdominal ganglion. The recording medium contained $50 \mathrm{~mm}$ tetraethylammonium chloride (TEA), which at these concentrations primarily blocks $\mathrm{K}^{+}$currents other than the serotonin-sensitize $\mathrm{K}^{+}$current $\left(\mathrm{I}_{\mathrm{K}, \mathrm{s}}\right)$, to facilitate measurement of the action potential duration and maximize the contribution of $I_{K, S}$ to action potential duration. The US was a brief $(1 \mathrm{sec})$ focal puff of serotonin (5-HT) that we applied directly onto the sensory neuron cluster via pressure ejection from a nearby

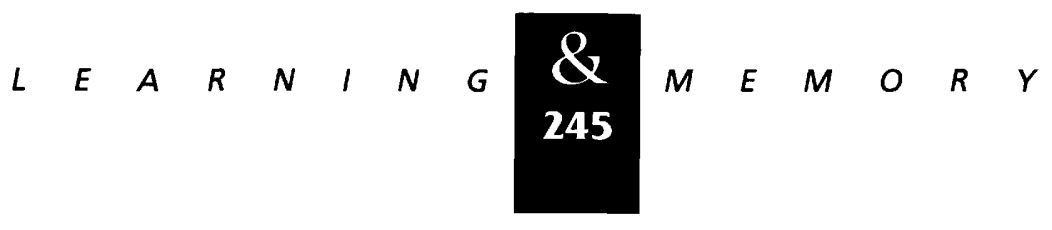


electrode. The electrode contained $10^{-4}$ M 5-HT, plus $1 \%$ fast green to aid visualization of $5 \cdot \mathrm{HT}$ application. The bath perfusion remained on during and after 5-HT delivery; visual inspection suggested that 5-HT had mostly cleared within 1 sec following offset of application. 5-HT delivery occurred either $0.5 \mathrm{sec}$ after (forward pairing) or 1.5 sec before (backward pairing) a spike train CS ( 10 $\mathrm{Hz}$, six spikes) in the sensory neuron. These experiments were performed with a counterbalanced ABA design. Each experimental series consisted of three pretraining tests (delivered at 30 sec intervals) in which a single action potential was elicited in the sensory neuron; a 3.5-min rest followed by one training trial (spike train CS with 5-HT US); and three post-training tests delivered at 30-sec intervals. The first training series was randomly chosen to be either a backward or forward condition; subsequent training series occurred 15 min apart.

Electrophysiological data were analyzed offline on a computer-based system (Hilal Associates) that calculated the amplitude and initial slope of the PSP, the amplitude and duration of the sensory neuron action potential, and the amplitude of the AHP. Action potential duration was measured by computer at $30 \%$ of maximum amplitude. Sensory neuron membrane potential during training trials was measured by hand from chart recordings.

Statistical analyses for PSP slope and amplitude and AHP amplitude were performed using measurements from the two trials immediately before and after training. For each sensory cell, the post-training scores were normalized to the mean of that cell's scores on the two pretraining trials in the same training series. The normalized scores were then averaged across the two cells and training series for each experimental condition so that each preparation contributed one difference score (e.g., forward pairing compared with backward pairing) to the overall group analysis. There were no significant differences between experimental conditions in the mean values for any dependent variable prior to training (for 0.5 -sec forward vs. 0.5 -sec backward experiments, $n=14$ : PSP amplitude, $5.83 \pm 1.20 \mathrm{mV}$ vs. $5.90 \pm 1.01 \mathrm{mV}$; PSP slope, $0.80 \pm 0.20 \mathrm{mV} / \mathrm{msec}$ vs. $0.88 \pm 0.20 \mathrm{mV} / \mathrm{msec}$ AHP amplitude, $5.74 \pm 0.61 \mathrm{mV}$ vs. $4.86 \pm 0.48 \mathrm{mV}$; for 0.5-sec forward vs. 5-sec forward experiments, $n=20: \quad$ PSP amplitude, $6.33 \pm 0.61 \mathrm{mV}$ vs. $6.42 \pm 0.71 \mathrm{mV}$; PSP slope, $0.65 \pm 0.09 \mathrm{mV} / \mathrm{msec}$ vs $0.76 \pm 0.12 \mathrm{mV} / \mathrm{msec}$ ). Reproducible measure- ments of the time to peak PSP amplitude were difficult to obtain in many experiments, so these data were not analyzed further. In spike-width experiments $(n=19)$, data for each cell were normalized to the mean of that cell's pretraining scores for the same training series; mean pretraining scores were not significantly different for the forward pairing condition (8.7 $\pm 1.4 \mathrm{msec})$ and backward pairing condition $(7.7 \pm 1.2 \mathrm{msec})$. Significance was evaluated using two-tailed $t$-tests for related means, except where indicated. All data are expressed as means \pm standard error of the mean (s.E.M.).

\section{Results}

\section{SHORT FORWARD PAIRINGS DIFFERENTIALLY \\ FACILITATE SYNAPTIC TRANSMISSION AT SENSORIMOTOR CONNECTIONS}

To investigate possible cellular mechanisms for the temporal specificity of classical conditioning, we first examined synaptic plasticity at connections of siphon sensory neurons induced by neural analogs of behavioral training at three critical time points of the ISI function. In separate experiments, we compared the consequences of a 0.5 -sec forward pairing condition (in which the CS precedes the US) that produces optimal behavioral conditioning with the consequences of two procedures that do not produce conditioning-a 0.5 sec backward pairing condition (in which US precedes CS) and a 5-sec forward pairing condition (Hawkins et al. 1986).

We found that 0.5 -sec forward pairings of a sensory neuron spike train CS and tail nerve shock US produced significantly greater synaptic facilitation than either the backward pairing condition or the 5-sec forward pairing condition, indicating that both the temporal order and proximity of the stimuli were important. Compared with backward pairings, forward pairings produced a significantly greater increase in the amplitude of the PSP [88 $\pm 14 \%$ increase vs. $50 \pm 11 \%$ increase, $n=14$, $t(13)=3.52, P<0.01]$ (Fig. 1). Forward pairing also produced a significantly greater increase in the initial slope of the PSP [ $70 \pm 14 \%$ increase vs. $37 \pm 9 \%$ increase, $t(13)=2.65, P<0.02]$. As described in more detail in the discussion, below, changes in PSP slope reflect one of the two cellular processes underlying facilitation. The PSP slope measurements also provided an independent measure of facilitation that was relatively free of pos-

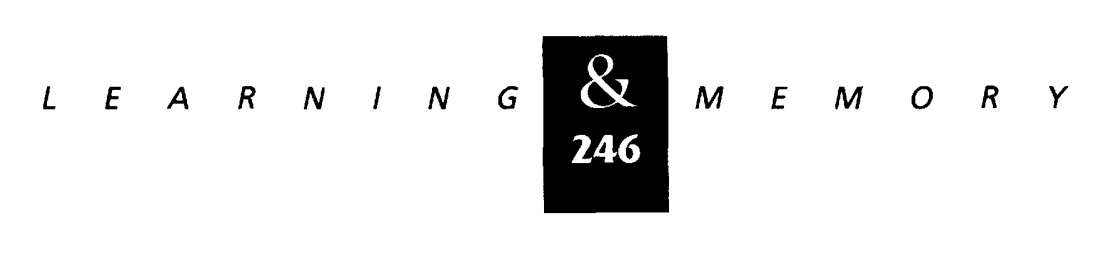


sible contributions from polysynaptic inputs. These temporally specific changes in PSP amplitude and slope (i.e., the difference between effects of forward and backward pairings) were highly correlated for individual preparations $(r=0.82$, $P<0.0001$ ) (Fig. 1D), suggesting that the two measures were mechanistically related.

Similar results were obtained in experiments comparing 0.5 -sec forward pairing with 5 -sec forward pairing (Fig. 2): The 0.5-sec forward pairing condition produced significantly greater enhancement of PSP amplitude than did the 5-sec forward pairing condition $[23 \pm 7 \%$ increase vs. $7 \pm 7 \%$ increase, $t(19)=2.54, P<0.02]$ and a marginally significant difference in PSP slope changes [25 $\pm 10 \%$ increase vs. $4 \pm 6 \%$ increase, $t(19)=2.02, P<0.05$ one-tailed]. Temporally specific changes in PSP amplitude and slope were again well correlated $(r=0.72, P<0.001)$.

The absolute amount of facilitation in these experiments was somewhat different from that in the experiments comparing forward and backward pairing, perhaps as a result of slightly different USs. We also note that some facilitation occurred in both the backward pairing and 5-sec forward pairing conditions; this facilitation may have resulted from small effects of pairing in these temporal conditions or from nonassociative effects [e.g., CS-induced post-tetanic potentiation (PTP) or US-induced heterosynaptic facilitation]. More importantly, however, the greater facilitation that occurred following 0.5 -sec forward pairings rela-
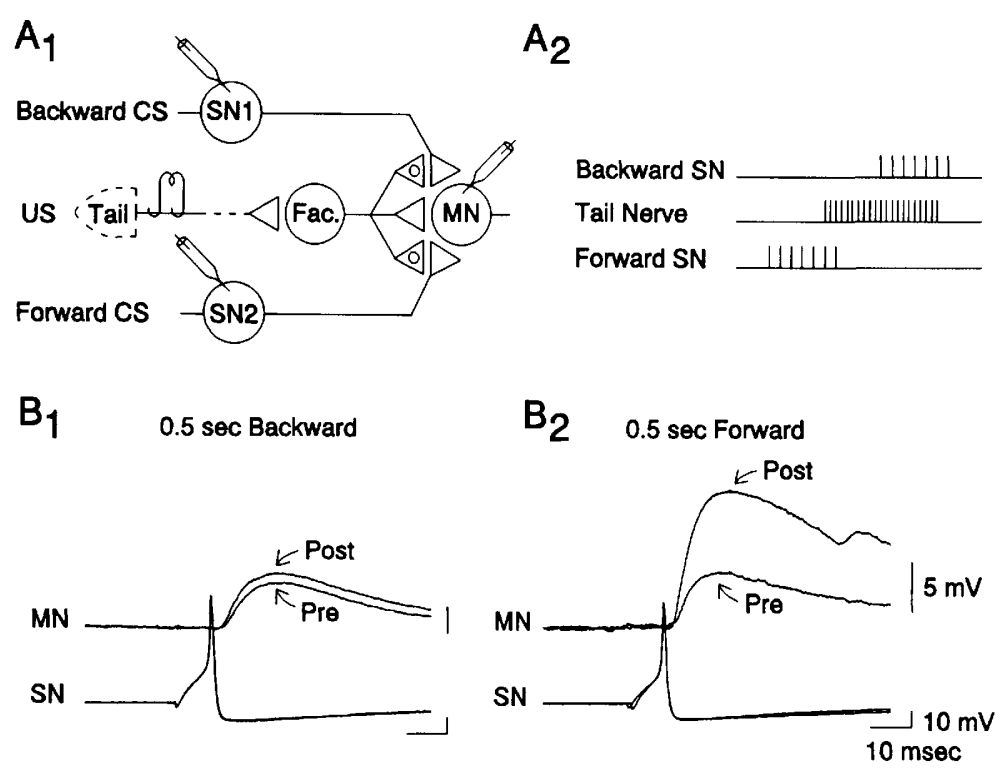

C

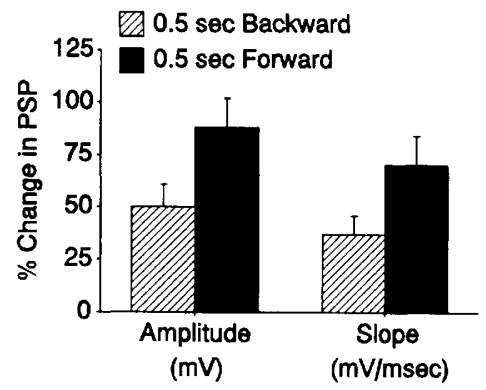

D

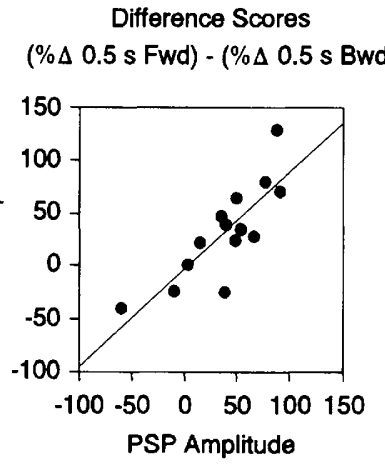

Figure 1: Forward pairing produces greater synaptic facilitation than does backward pairing. $\left(A_{1}\right)$ Experimental arrangement and $\left(A_{2}\right)$ training protocol for comparing synaptic facilitation produced by $0.5-\mathrm{sec}$ forward CSUS pairings and 0.5 -sec backward pairings. Intracellular recordings were obtained from two different sensory neurons (SN1 and SN2) and a siphon motor neuron ( $\mathrm{MN}$ ) or interneuron to which they both projected. The PSP elicited in the motor neuron was tested before and after training. For one sensory neuron, the forward paired cell, the spike train $\mathrm{CS}(10 \mathrm{~Hz}$, seven spikes) used in training began $0.5 \mathrm{sec}$ before the tail (pedal) nerve shock US (10-20 Hz, $1.0 \mathrm{sec})$, which activates facilitatory interneurons (Fac.). For the other sensory neuron (the backward paired cell), the spike train CS began $0.5 \mathrm{sec}$ after US onset. Thus, the only difference between the treatment of the two sensory neurons was the temporal relationship of the spike activity to the tail nerve shock. The tail (broken lines) is depicted for illustrative purposes but was not included in actual experiments. (B) Representative example from one preparation showing greater synaptic facilitation following forward CS-US pairings $\left(B_{2}\right)$ than following backward CS-US pairings $\left(B_{7}\right)$. Top traces illustrate synaptic potentials elicited in a single siphon motor neuron ( $\mathrm{MN}$ ) by an action potential in the sensory neuron $(\mathrm{SN})$ before and after each type of training. (C) Group data for mean changes in PSP amplitude and

PSP slope following forward and backward pairing. Both measures were preferentially enhanced for the 0.5 -sec forward training condition. Error bars indicate S.E.M. for this and subsequent figures. (D) Differential changes in PSP amplitude and PSP slope are correlated for the 0.5-sec forward vs. 0.5-sec backward training conditions. Difference scores for each preparation represent the percent change in PSP amplitude (or PSP slope) following forward pairing, minus the percent change following backward pairing.

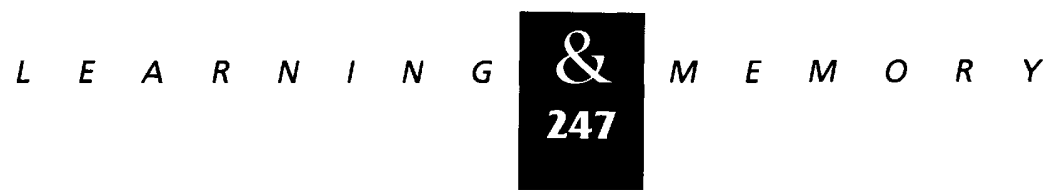


Figure 2: Forward pairing at short ISIs produces greater synaptic facilitation than forward pairing at long ISIs. (A) Representative traces from one preparation. The spike train CS was paired with tail nerve shock US at a 5.0-sec forward ISI for one sensory neuron $\left(A_{1}\right)$ and at a 0.5 -sec forward ISI for the other sensory neuron $\left(A_{2}\right)$, which showed enhanced facilitation. ( $S N$ ) sensory neuron; $(\mathrm{MN})$ motor neuron. (B) Group data indicating greater facilitation of mean PSP amplitude and PSP slope in the 0.5 -sec forward pairing condition. (C) Differential changes in PSP amplitude and PSP slope are correlated for the 0.5 -sec forward vs. $5.0-\mathrm{sec}$ forward training conditions. Difference scores represent the percent change in PSP amplitude (or PSP slope) following $0.5-\mathrm{sec}$ forward pairing, $\mathrm{mi}-$ nus the percent change following 5.0 -sec forward pairing.
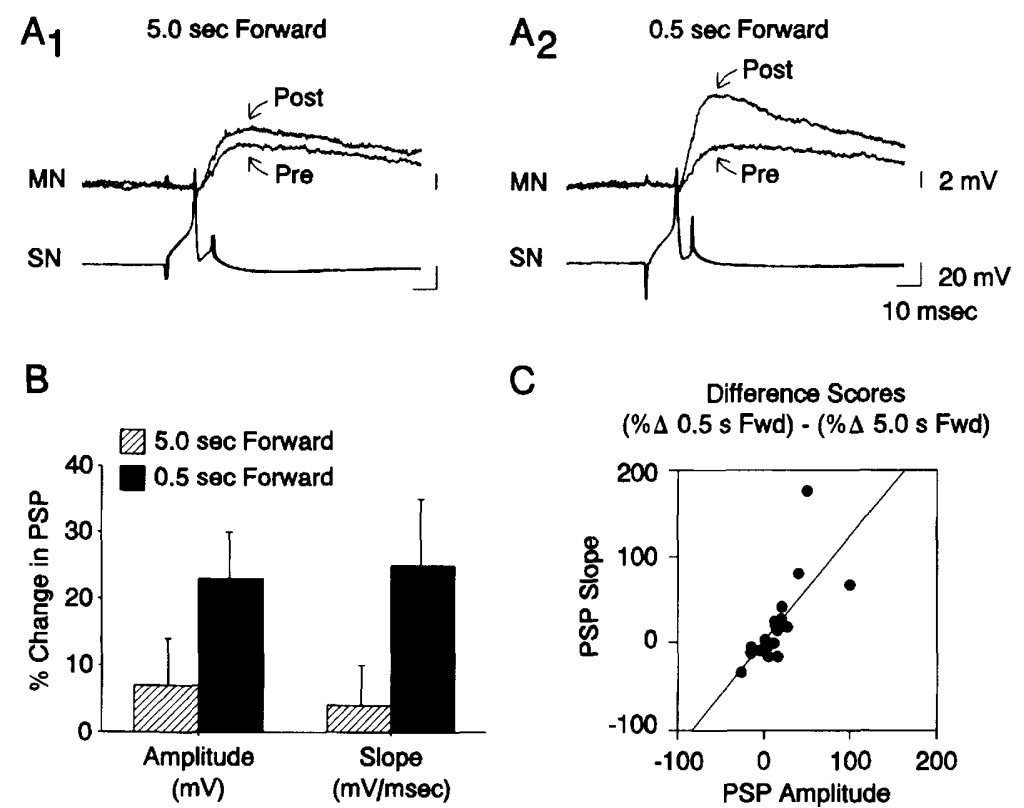

C
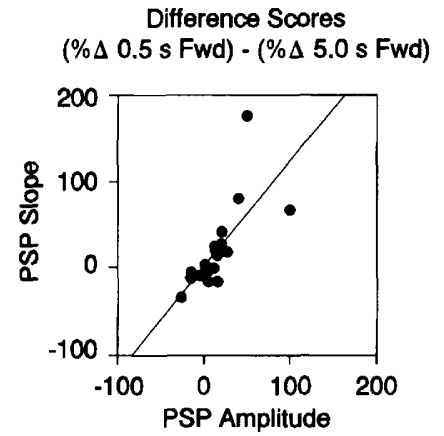

tive to the other training conditions in the same preparations suggests that activity-dependent enhancement of synaptic facilitation at sensory neuron connections could provide a neural substrate for the temporal specificity of conditioning evident in behavior.

\section{EFFECTS ON SENSORY CELL MEMBRANE POTENTIAL DURING TRAINING TRIALS}

Sensitizing stimuli can produce both depolarization and hyperpolarization in Aplysia mechanosensory cells (Hawkins et al. 1981a; Walters and Byrne 1983b, 1985; Walters et al. 1983; Mackey et al. 1987). In principle, these actions could influence CS-evoked events during the training trials per se, especially for the backward paired cell whose CS entirely follows the US. In particular, US-induced inhibition of the backward CS sensory cell might diminish $\mathrm{Ca}^{2+}$ influx during the CSevoked action potential activity and thereby reduce activity-dependent enhancement of facilitation in backward pairing compared with forward pairing (Mackey et al. 1987).

To examine this and related possibilities and to define more accurately the time course of depolarization and inhibition relative to our CSs, we first measured the effects of the US on the membrane potential of sensory cells receiving 5-sec forward paired training. These sensory neurons were inactive during the time period immediately before and after the US, thereby allowing effects of the US to be examined in isolation from the effects of the CS spike train. We found that the US evoked an initial depolarization $[1.5 \pm 0.2 \mathrm{mV}, t(19)=$ $6.17, P<0.001]$ that declined to near baseline values by $300 \mathrm{msec}$ and was transformed into a hyperpolarization at $500 \mathrm{msec}$ post-US onset $[-2.6 \pm 0.72 \mathrm{mV}, t(19)=3.6, P<0.01]$ and thereafter, the time the backward CS would have been presented on a 0.5-sec backward training trial (Fig. 3A).

Because these measurements were taken from different preparations involving slightly different USs, we cannot conclude that the same amount of inhibition was necessarily present in our backward paired cells. To examine this issue more directly and to explore possible interactions of these membrane-potential shifts with CS-evoked spike activity, we also measured the membrane potential during training trials in cells receiving 0.5 -sec forward and 0.5 -sec backward pairing (Fig. 3B). Confirming the general pattern found above, the backward paired cells exhibited an initial peak depolarization 100-300 msec following US onset [ $1.90 \pm 0.77$ $\mathrm{mV}, t(13)=2.46, P<0.05]$, which then declined significantly to near baseline values by the start of the backward CS. It was not possible to measure directly the resting membrane potential during the backward CS, because of the CS-elicited spike activity. To infer US-evoked effects, we therefore compared the membrane potential values for the

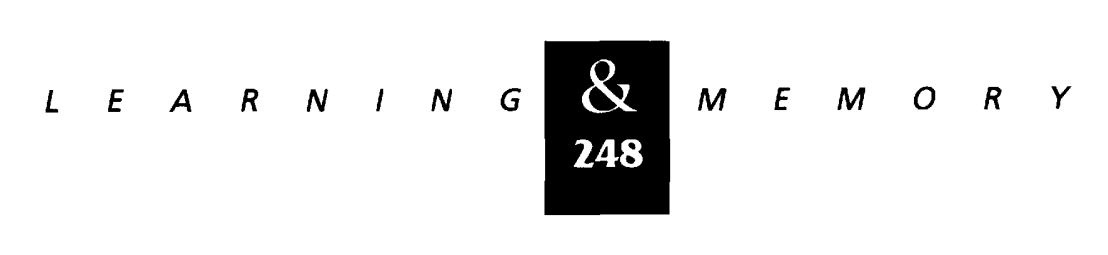


A

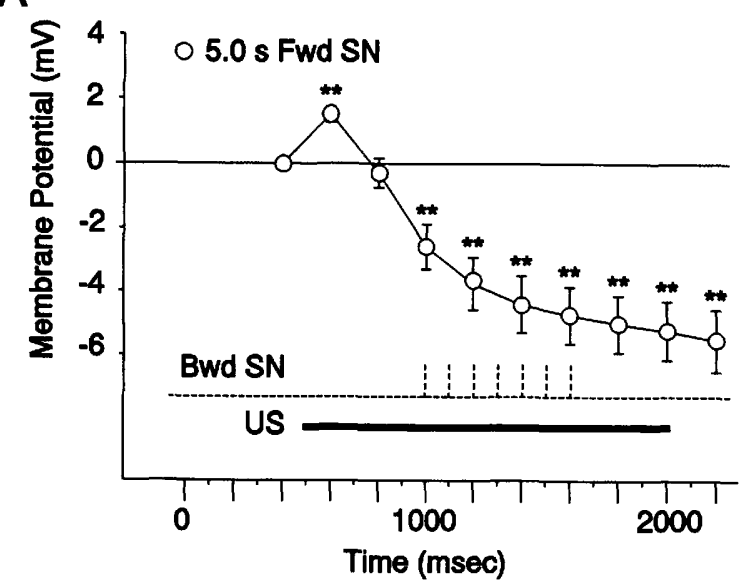

B

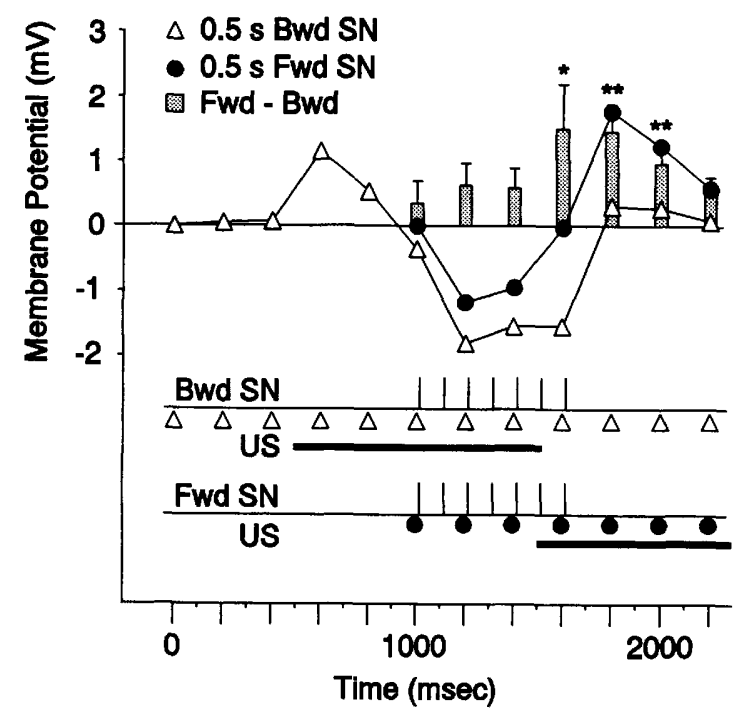

backward paired cell during the backward CS with the membrane potentials for the 0.5 -sec forward paired cell during the forward CS. We found a forward-backward difference that increased during the CS period, becoming statistically reliable by the end of the CS period and for the period thereafter. These forward-backward differences probably arose from a US-induced depolarization in the forward paired cell at the end of the forward CS and from inhibition in the backward paired cell during the backward CS.

\section{SHORT FORWARD PAIRINGS DIFFERENTIALLY MODIFY SENSORY NEURON ACTION POTENTIAL SHAPE}

5-HT and sensitizing stimuli reduce particular $\mathrm{K}^{+}$currents in Aplysia sensory cells $\left(\mathrm{I}_{\mathrm{K}, \mathrm{s}}\right.$ and $\left.\mathrm{I}_{\mathrm{K}, \mathrm{v}}\right)$,
Figure 3: Effects of training trials on sensory neuron membrane potential. Data are from the same preparations used to examine synaptic facilitation. (A) In the $5.0-\mathrm{sec}$ forward paired cells (which were inactive in the period immediately preceding and following the US), the US evoked an initial depolarization followed by a hyperpolarization. Time of US presentation is indicated by thick horizontal line below the plot. Also indicated for reference is the time that the 0.5 -sec backward CS would have been presented in other experiments (dashed spikes). Hyperpolarization is clearly present at these time points. $\left[\left(^{* *}\right) P<0.01\right.$, two-tailed t-tests.] (B) Membrane potentials of sensory cells receiving $0.5-\mathrm{sec}$ backward pairing and 0.5 -sec forward pairing. To facilitate comparisons of measurements during the CSs, the data for the cells trained with forward pairings (Fwd SN) have been shifted rightward so that the CS period is aligned with the CS period for cells trained with backward pairings (Bwd $\mathrm{SN}$ ). Histogram bars indicate the difference in membrane potential between the two cells $\left[\left({ }^{*}\right) P<0.05 ;\left(^{* *}\right) P<0.01\right.$, two- tailed t-tests]. The schematic below indicates the pattern of CS activity (spikes) and US stimulation (thick horizontal line) delivered to each cell, plus the times at which measurements were obtained. During the CS period, membrane potential was measured just prior to the onset of individual action potentials in the spike train, at which point the AHP from the preceding action potential had not completely ended, so US effects must be interpreted with respect to these CS-related hyperpolarizations. A reliable difference between the membrane potential in the forward paired cells and backward paired cells emerged by the end of the CS period and thereafter, which appears to reflect a combination of US-induced depolarization in the forward paired cell and US-induced hyperpolarization in the backward paired cell.

which in turn broadens the action potential and contributes to facilitation of transmitter release from sensory neurons (Klein and Kandel 1980; Klein et al. 1982; Siegelbaum et al. 1982; Belardetti et al. 1986; Hochner et al. 1986a; Baxter and Byrne 1989, 1990; Goldsmith and Abrams 1992; Hochner and Kandel 1992; Sugita et al. 1992; Eliot et al. 1993). The action potential broadening and the changes in ionic currents, like synaptic facilitation, can be enhanced in a pairing-specific manner (Hawkins et al. 1983; Hawkins and Abrams 1984; Eliot et al. 1994). We therefore asked, Do the sensory neurons also undergo temporally-specific changes in ionic currents and action potential shape that could contribute to the ISI function for synaptic facilitation?

Our experiments on synaptic facilitation used relatively long $(>10 \mathrm{msec})$ intracellular com-

$$
\begin{array}{llllllll}
L & E & A & R & N & I & N & G \\
\mathbf{2 4 9} & \mathbf{Q} & M & E & M & O & R & Y
\end{array}
$$


mands to evoke action potentials in the sensory neurons reliably. Because these command pulses overlapped the action potential peak, it was not possible to measure the action potential duration accurately in these experiments. However, previous work has indicated that activation of L29, a facilitatory interneuron that is activated by tail shock and produces both spike broadening and facilitation at sensory neuron connections (Hawkins 1981; Hawkins et al. 1981b; Hawkins and Schacher 1989), also decreases the sensory neuron AHP (Hawkins et al. 1981b). As a first step toward examining whether temporally specific changes in sensory neuron currents accompanied the observed changes in synaptic strength, we therefore asked whether forward pairing compared with backward pairing differentially reduced the sensory neuron AHP, measured in the same preparations in which we compared effects of forward and backward pairings on synaptic plasticity. (In the 0.5-sec forward vs. 5-sec forward experiments, we used slightly longer current pulses that extended into the AHP itself, preventing a reliable measurement of the AHP in those experiments.) We found that following the 0.5 -sec forward pairing condition, sensory neurons exhibited a significantly greater decrease in AHP amplitude than following the backward pairing condition $[4 \pm 2 \%$ decrease vs. $4 \pm 3 \%$ increase, $t(13)=2.88, P<0.02$ ] (Fig. 4). Although the AHP has not been well analyzed in these cells, the changes in the AHP most likely arose from alterations in $\mathrm{K}^{+}$currents $\left(\mathrm{I}_{\mathrm{K}, \mathrm{S}}, \mathrm{I}_{\mathrm{K}, \mathrm{V}}\right.$, and $\left.\mathrm{I}_{\mathrm{K}, \mathrm{Ca}}\right)$ that are modulated by 5 -HT and sensitizing stimuli (Klein and Kandel 1980; Klein et al. 1982; Siegelbaum et al. 1982; Belardetti et al. 1986; Hochner et al. 1986a; Baxter and Byrne 1989, 1990; Walsh and Byrne 1989; Goldsmith and Abrams 1992; Hochner and Kandel 1992; Sugita et al. 1992). These findings demonstrate that temporally specific changes in sensory neuron currents had indeed occurred and provide initial evidence that the associative mechanisms underlying temporally specific synaptic facilitation involved processes intrinsic to the sensory neurons themselves.

To examine the effects of different ISIs on sensory neuron action potentials and currents more directly, we conducted an additional set of experiments explicitly designed to compare changes in action potential duration in individual sensory neurons following forward and backward pairing conditions. The isolated abdominal ganglion was bathed in $50 \mathrm{~mm}$ TEA in order to prolong the ac-
$A_{1} 0.5$ sec Backward $\quad A_{2} 0.5$ sec Forward

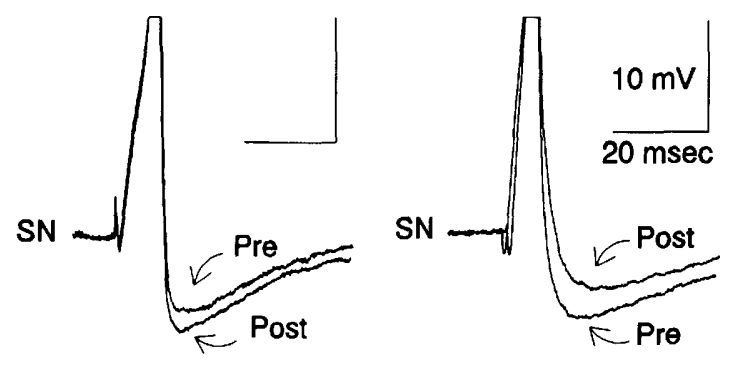

B

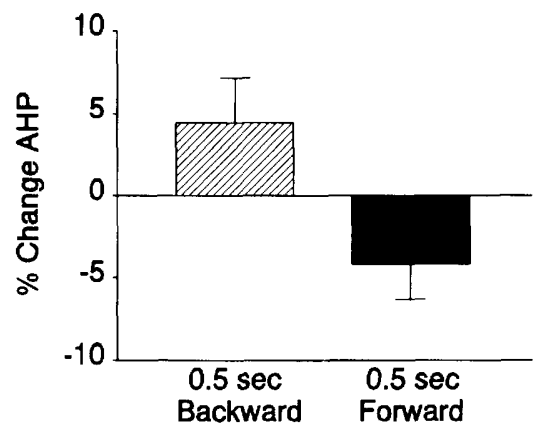

Figure 4: Forward pairing differentially reduces the sensory neuron AHP. (A) Examples of AHP changes in two sensory neurons ( $\mathrm{SN}$ ) following 0.5 -sec backward pairing $\left(A_{1}\right)$ and $0.5-\mathrm{sec}$ forward pairing $\left(A_{2}\right)$. The AHP increased following backward pairing but decreased following forward pairing. The tops of the action potentials have been electronically clipped to allow illustration at higher gain. Traces are aligned at the peaks of the action potentials (not shown). (B) Group data, from the same preparations used for PSP data.

tion potential and maximize the contribution of $\mathrm{I}_{\mathrm{k}, \mathrm{s}}$. In addition, to simplify and restrict the actions of the US, we substituted for the tail nerve shock US a brief ( $1 \mathrm{sec}$, plus $\sim 1$-sec washout) puff of $10^{-4}$ M 5-HT, a facilitatory transmitter normally released by tail shock (Brunelli et al. 1976; Glanzman et al. 1989; Hawkins 1989; Mackey et al. 1989), which we applied directly via microperfusion onto the sensory neuron cluster. 5-HT delivery occurred either $0.5 \mathrm{sec}$ after (forward pairing) or $1.5 \mathrm{sec}$ before (backward pairing) a spike train $\mathrm{CS}$ in the sensory neuron. We found that increases in spike duration were greater following forward pairing than backward pairing $[51 \pm 9 \%$ increase vs. $36 \pm 9 \%$ increase, $n=19, t(18)=2.15, P<0.05$ ] (Fig. 5). Because spike broadening in siphon sensory cells prolongs $\mathrm{Ca}^{2+}$ influx and thereby enhances transmitter release, these temporally spe-

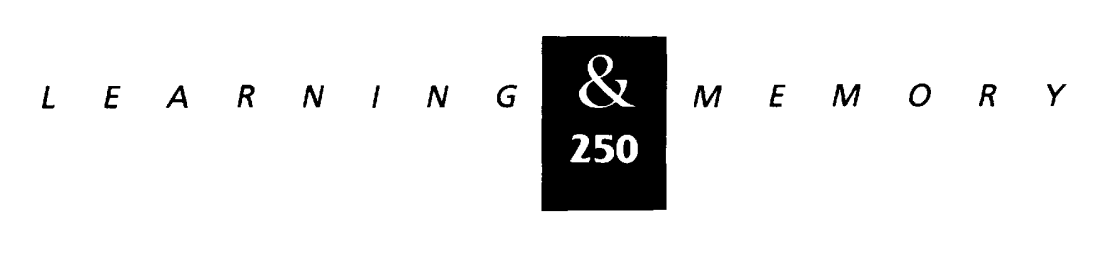


$A_{1}$

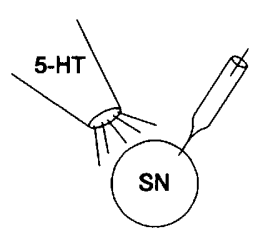

$A_{2}$

Backward

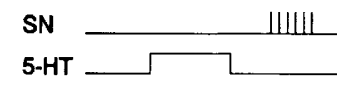

Forward
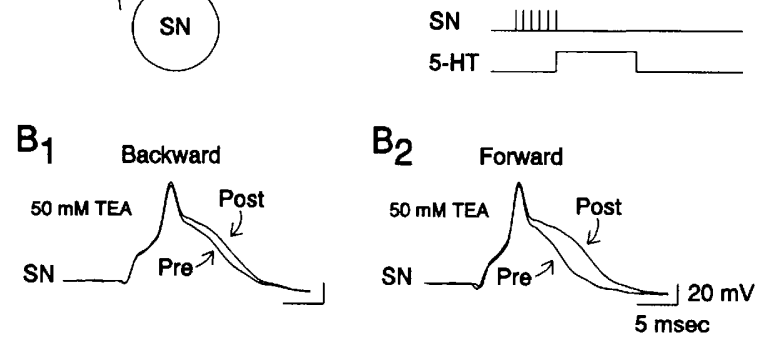

C

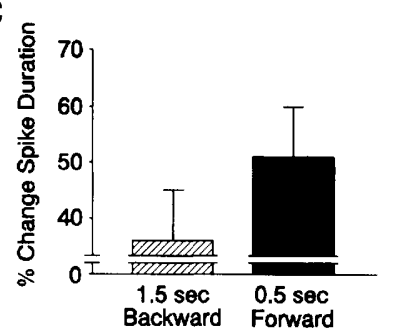

Figure 5: Forward pairing produces greater spike broadening than does backward pairing. $\left(A_{1}\right)$ Experimental arrangement and $\left(A_{2}\right)$ training paradigm. A 10$\mathrm{Hz}$ train of six spikes in the sensory neuron (SN) was paired with a brief ( $1 \mathrm{sec}$, plus $\sim 1$-sec washout) puff of $10^{-4}$ M 5-HT onto the sensory cell cluster. Each cell was examined under both a forward pairing condition (in which the spike train CS preceded the serotonin puff US by $0.5 \mathrm{sec}$ ) and a backward pairing condition (in which the spike train followed the onset of the serotonin puff by $1.5 \mathrm{sec}$ ). (B) Representative example from a single cell showing greater spike broadening following forward pairing $\left(B_{2}\right)$ than following backward pairing $\left(B_{1}\right)$. (C) Group data illustrating greater spike broadening following forward pairing.

cific changes in sensory neuron spike duration could contribute to the temporally specific enhancement of synaptic potential amplitude.

To examine possible effects of 5-HT applications on sensory cell membrane potentials, we measured the membrane potential during the interval from US onset to CS onset in the backward pairing condition. We found that these brief, restricted 5-HT applications (unlike tail nerve shock) did not produce any significant depolarization or hyperpolarization of sensory cells $(P>0.10)$. These results suggest that forwardbackward differences in spike broadening were not the result of modulation of sensory cell membrane potential by interneurons activated by $5-\mathrm{HT}$.

\section{Discussion}

\section{ACTIVITY-DEPENDENT ENHANCEMENT OF PRESYNAPTIC FACILITATION EXHIBITS TEMPORAL SPECIFICITY}

The present studies demonstrate that facilitation at the connections between Aplysia siphon sensory cells and their targets exhibits, and could therefore contribute to, the temporal specificity evident on a behavioral level in classical conditioning of the siphon-withdrawal response. In brief, short forward pairings of the neural CS and US produced more pronounced neuronal changes than did either long forward pairings or backward pairings, as evidenced in several physiological measures: the amplitude and the slope of the EPSP at sensorimotor connections, the amplitude of sensory neuron AHPs, and the duration of sensory neuron action potentials. Because these same cellular measures have been found previously to be modulated by US-alone presentations, this associative mechanism appears to involve an amplification of the US-evoked neuromodulatory effects by spike activity in the sensory neurons (i.e., activitydependent enhancement of presynaptic facilitation). Thus, the temporal specificity of associative conditioning, like the pairing specificity, incorporates and elaborates on the same basic facilitatory process involved in nonassociative learning (sensitization), rather than utilizing an entirely separate mechanism. The present experiments extend results of previous studies on pairing specificity by demonstrating that activity-dependent enhancement of presynaptic facilitation is also sensitive to both the order and temporal proximity of the cellular signals for the CS and US. Activity-dependent enhancement of presynaptic facilitation may therefore provide a neural basis for why, on a behavioral level, the CS and US must occur closely together in time and why the CS must precede the US for associative learning to occur.

\section{DUAL MECHANISMS OF FACILITATION}

Presynaptic facilitation in sensory neurons can arise from two separate processes: first, the suppression of serotonin-sensitive $\mathrm{K}^{+}$currents $\left(\mathrm{I}_{\mathrm{K}, \mathrm{S}}\right.$ and $I_{K, V}$ ), which prolongs the duration of the sensory neuron action potential and thereby enhances calcium influx and transmitter release (Klein and Kandel 1978, 1980; Klein et al. 1982;

$$
\text { n...... 通 }
$$


Siegelbaum et al. 1982; Belardetti et al. 1986; Hochner et al. 1986a; Baxter and Byrne 1989, 1990; Goldsmith and Abrams 1992; Hochner and Kandel 1992; Sugita et al. 1992; Eliot et al. 1993); and a second process that is independent of ionic conductance changes and reflects a direct action on transmitter mobilization or release (Hochner et al. 1986b; Dale et al. 1988; Braha et al. 1990; Sacktor and Schwartz 1990; Goldsmith and Abrams 1991; Ghirardi et al. 1992; Klein 1993, 1994; Eliot et al. 1994). Our results suggest that both of these processes are modulated in an associative, temporally specific manner.

We found direct evidence that forward pairing of sensory cell spike activity and 5-HT produces greater action potential broadening than does backward pairing. Our recordings were made from the sensory cell somata, but changes in somatic currents are believed to correlate with similar changes occurring in synaptic terminal regions (Belardetti et al. 1986; Hochner et al. 1986a; Eliot et al. 1993). The changes in action potential duration that we observed most likely contribute to the enhancement of synaptic strength, suggesting that temporally specific synaptic facilitation involves presynaptic mechanisms intrinsic to the sensory cells.

In normal ASW, spike broadening is believed to result from reductions in both $I_{K, S}$ and $I_{K, V}$. However, our experiments examining action po- tential broadening were conducted in $50 \mathrm{~mm}$ TEA, which would be expected to suppress most of the contribution of $I_{K, V}$ to action potential repolarization. The differential effects we observed under these conditions thus suggest that $I_{K, S}$ can be reduced in a temporally specific manner, in addition to a pairing-specific manner as shown previously (Hawkins and Abrams 1984). Reduction of $I_{K, S}$ can contribute to increased excitability of sensory cells as well as action potential broadening (Klein et al. 1986; Baxter and Byrne 1990; Mercer et al. 1991; Goldsmith and Abrams 1992; Byrne et al. 1993), suggesting that sensory cells might also show temporally specific increases in excitability, though we did not measure this in our experiments. Because modulation of $I_{K, S}$ and $I_{K, V}$ share many mechanistic steps, it is also quite possible that modulation of $I_{K, V}$ exhibits temporal specificity and could therefore have contributed to the differential synaptic facilitation we observed in normal ASW.

We did not test for potential changes in action potential duration in normal ASW for technical reasons, so we do not know how much temporally specific spike broadening would occur in normal ASW. However, we did observe a temporally specific reduction in sensory neuron AHP amplitude in normal ASW. These observations indicate that at least some temporally specific changes in sensory neuron currents and action potential shape are not an artifact of TEA recording solutions and provide further evidence for intrinsic presynaptic changes in the sensory neurons. The $\mathrm{K}^{+}$currents mediating the AHP in siphon sensory cells have not been explored well, and the functional significance of reduced AHPs is unclear. It is possible that reduced AHPs would minimize deinactivation of fast $\mathrm{K}^{+}$currents and thus increase the frequency of action potential firing (Connor and Stevens 1971). Alternatively, AHP reductions may have little functional impact on their own but may nonetheless reflect mechanisms involved in modulation of $\mathrm{K}^{+}$ conductances with other important consequences, such as action potential broadening. In either case, the fact that AHP reductions are produced by facilitatory interneurons along with the other modulatory actions, spike broadening and synaptic facilitation (Hawkins 1981; Hawkins et al. 1981b), suggests that they may serve as a useful readout of sensory neuron changes. Reductions of the AHP in hippocampal pyramidal cells have also been reported following classical conditioning of the rabbit eye-blink/nictitating membrane response (Disterhoft et al. 1986).

Although both spike-width-dependent and spike-width-independent mechanisms of facilitation increase PSP amplitude, only the latter process is believed to increase the rate of rise of the PSP (Hochner et al. 1986b). The temporally specific changes in PSP slope that we observed provide the first evidence that this second set of processes, implicated previously in nonassociative sensitization and dishabituation, may be modulated in an associative manner as well. The strong correlation between changes in the PSP amplitude and PSP slope suggests further that this second facilitatory process contributes importantly to the changes in PSP amplitude, or that the two facilitatory processes arise from a common molecular signal. Other experiments indicate that spontaneous transmitter release is not changed in a pairingspecific manner (Eliot et al. 1994), suggesting that associative changes occurring in the second process may engage primarily mechanisms involved in coupling of the action potential with release.

It seems unlikely that the differential effects

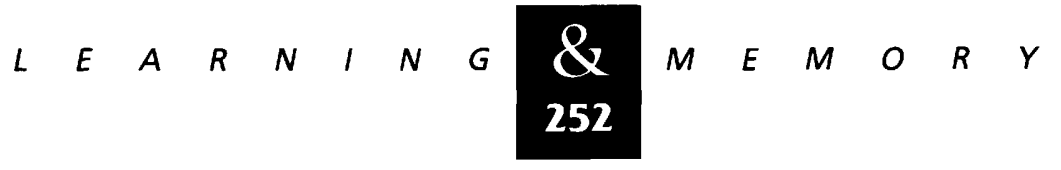


on synaptic transmission we observed with short forward pairings can be accounted for by a generalized postsynaptic change, because we utilized two different inputs onto a common postsynaptic cell. Our experiments do not explicitly rule out more specific postsynaptic contributions from the siphon motor neurons, though other work on activity-dependent facilitation has found no postsynaptic change in transmitter receptor sensitivity (Eliot et al. 1994).

Taken together, our results suggest that intrinsic sensory cell changes in both the first process of facilitation $\left(\mathrm{K}^{+}\right.$current suppression and action potential broadening) and some aspect of the second process (e.g., spike/release coupling) contribute to the ISI function for synaptic facilitation.

\section{CELLULAR AND CIRCUIT MECHANISMS OF TEMPORAL SPECIFICITY}

What mechanisms mediate temporal specificity at the molecular level? One attractive possibility (Hawkins et al. 1983; Walters and Byrne 1983a; Abrams 1985; Occor et al. 1985; Yovell and Abrams 1992) is that temporal specificity arises from the properties of a calcium/calmodulin-sensitive form of adenylate cyclase, the enzyme that produces CAMP in sensory cells during facilitation. cAMP is an important second messenger involved in the induction of several aspects of sensory cell plasticity in sensitization and conditioning, including spike broadening and synaptic facilitation (Brunelli et al. 1976; Klein and Kandel 1978; Braha et al. 1990; Byrne et al. 1993). According to this view, $\mathrm{Ca}^{2+}$ entry into sensory neurons during spike activity (produced by the CS) can prime the calcium-sensitive cyclase and thereby enhance the cAMP cascade and the resultant synaptic facilitation triggered by the US. Long forward CS-US intervals are thus relatively ineffective in establishing conditioning because intracellular calcium levels decline toward baseline rather quickly, so that the calcium-triggered priming of the cyclase has declined by the time the US is delivered. Activation of the cyclase also displays a sequence requirement that may contribute to the differences between forward and backward pairing: Cyclase activation is greater when $\mathrm{Ca}^{2+}$ pulses precede rather than follow 5-HT (Yovell and Abrams 1992). The intrinsic properties of the cyclase, in conjunction with other intracellular signals, could thus contribute to the differential facilitation that we found to occur with short forward pairings relative to backward pairings.

Other mechanisms, such as network effects, may also contribute. In support of this possibility, we found differences in the membrane potential of sensory neurons during forward pairing and backward pairing trials, which appeared to arise from a US-induced depolarization during forward pairing and a US-induced hyperpolarization during backward pairing. During backward pairing in the intact animal, US-evoked inhibition might in principle decrease either the number of action potentials elicited by the CS (though this number was held constant in our physiological experiments) or the calcium entry per action potential and thereby diminish the associative enhancement that normally occurs. Similarly, in forward pairing, the short-latency US-evoked depolarization could in principle enhance sensory neuron activation or calcium influx. The effects of these changes in membrane potential on synaptic plasticity have not been tested, but our results document that the time course of inhibition and depolarization would allow such interactions to occur. Differential effects on membrane potential following paired training have previously been proposed to contribute to associative changes in Aplysia mechanosensory cells (Walters and Byrne 1983b; Walters et al. 1983; Mackey et al. 1987) as well as in Hermissenda Type $\mathrm{B}$ photoreceptors (Alkon 1979, 1980).

Taken together, these observations suggest that intrinsic molecular mechanisms (e.g., temporally asymmetrical sensitivity of the cyclase) and network processes (e.g., activation of inhibitory interneurons) could both play a role in the temporal specificity of activity-dependent enhancement of presynaptic facilitation. Although the present experiments do not permit definitive conclusions regarding the relative contributions of these mechanisms, the differential action potential broadening we observed with forward pairings compared with backward pairings involving restricted 5-HT applications suggests an important role for intrinsic processes. In these experiments, the brief, restricted 5-HT pulses we used did not hyperpolarize the sensory neurons, suggesting that differential effects were not due to 5-HT-elicited inhibition which blunted the effects of backward pairing. These data thus support the possibility that the temporal plasticity profile of activity-dependent facilitation [unlike that of Hebbian longterm potentiation (LTP), as described below] is

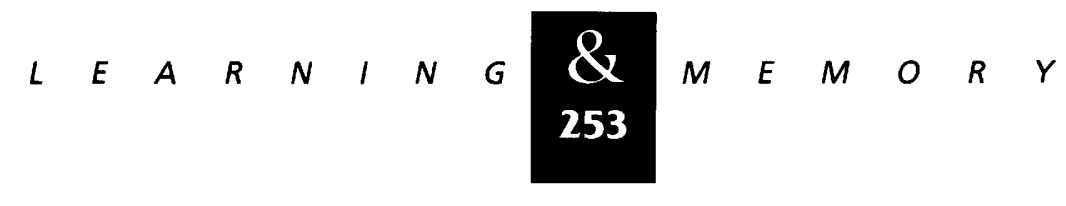


intrinsically asymmetrical (Yovell and Abrams 1992), with forward pairings being more efficacious than backward pairings. However, it is also possible that the differential spike broadening we observed instead reflects differences in the timing of the forward and backward CSs relative to the 5-HT pulse. In other work, the lack of a reliable difference between 0.5 -sec forward pairing and long (2.5-min) backward pairing of a spike train CS and 5-HT US in dissociated cell culture (Eliot 1991) might seem to suggest that network processes such as inhibition are necessary. However, those experiments employed a much stronger CS ( $20 \mathrm{~Hz}$, for $2 \mathrm{sec}$ ) that produces substantial PTP by itself, so that the previous treatment with 5-H'T could have enhanced the induction of PTP.

Irrespective of the precise contributions of the different intracellular and extracellular signals elicited by the CS and US, the present experiments indicate that activity-dependent facilitation is preferentially expressed following short forward pairings relative to either long forward pairings or backward pairings, and may thus contibute to both the CS-US sequence and proximity requirements of conditioning.

\section{COMPARISON WITH LTP AND LTD}

Certain other associative forms of synaptic modification also exhibit strict temporal constraints in the parameters for induction, though the particular requirements differ. In LTP in hippocampus, simultaneous activity in different inputs, or in the pre- and postsynaptic cells, produces optimal facilitation (Gustafsson and Wigström 1986; Kelso et al. 1986). Long-term depression (LTD) in cerebellum or hippocampus typically occurs following conjunctive stimulation with a slightly backward phase relationship (Ekerot and Kano 1989; Sejnowski et al. 1990; Chen and Thompson 1992), though cerebellar LTD may also be obtained using a $250-\mathrm{msec}$ forward interval (Chen and Thompson 1992). It is possible that these differences in temporal requirements are related to the different functions that these forms of plasticity serve. For example, hippocampal LTP has been implicated in "declarative" or "explicit" memory that can encode stimuli or events that occur simultaneously (Eichenbaum et al. 1992; Squire 1992), whereas activity-dependent facilitation contributes to "nondeclarative" or "implicit" classical conditioning, which often involves learn- ing about predictive relationships between events that may be separated in time.

In contrast with earlier results (Carew et al. 1984), Lin and Glanzman (1994a,b) have recently found that Aplysia sensorimotor connections in vitro exhibit Hebbian LTP, similar to that observed in hippocampus, and there is now some evidence suggesting Hebbian LTP may occur at sensorimotor connections in the ganglion as well (Cui and Walters 1994; P.M. Balaban, X. Fang, and G.A. Clark, unpubl.). Hebbian LTP is an attractive neural mechanism that could explain many associative features of conditioning (Hawkins et al. 1989; Glanzman 1994, 1995). However, it seems unlikely that a Hebbian mechanism alone can completely account for the temporal specificity of conditioning on a behavioral level or for all the results of the present studies.

One question for Hebbian LTP is how to explain the relatively weak effects of simultaneous and backward behavioral conditioning. Hebbian LTP is strongest with simultaneous coactivation of pre- and postsynaptic cells and diminishes comparably with delays in either a forward or backward direction (Glanzman and Lin 1994). To account for the differential effects of forward pairing compared with backward pairing, it is thus necessary to posit additional mechanisms, such as US-evoked inhibition during backward pairing (Glanzman and Lin 1994), as reported here. In conjunction with US-induced inhibition, Hebbian facilitation may have contributed to the enhanced synaptic facilitation we observed with short forward pairing using tail nerve shock US. However, we also found that forward pairing differentially increased the duration of sensory neuron action potentials when the US consisted of localized 5-HT applications, and it seems less likely that Hebbian mechanisms could explain these latter results, for several reasons. First, Hebbian mechanisms have not been shown to modulate spike duration. In contrast, both nonassociative and associative forms of spike broadening can occur in isolated sensory cells without postsynaptic targets (Eliot et al. 1994), where Hebbian mechanisms would not contribute. Second, in our experiments, 5-HT overlapped the backward CS but not the forward CS, and there was no detectable US-induced inhibition which might have diminished the effects of backward pairing; one might thus expect that Hebbian mechanisms would have induced comparable or greater spike broadening in the backward paired cell, in contrast with our actual results. Finally, the brief

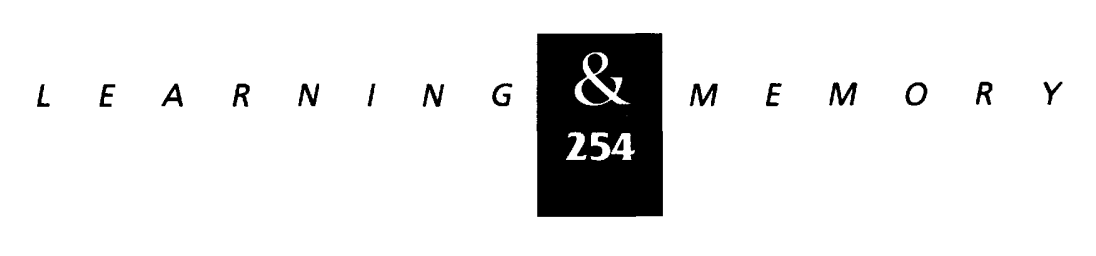


restricted 5-HT applications we used would produce relatively little depolarization of the postsynaptic targets, particularly in synaptic regions that are in the interior of the ganglion and were not directly exposed to 5-HT puffs; this would minimize possible Hebbian contributions. The differential spike broadening we observed is thus more likely to have resulted from activity-dependent facilitation.

It is important to emphasize that activity-dependent facilitation and Hebbian LTP are not mutually exclusive, and in fact may share certain steps in their underlying cellular and molecular substrates (Hawkins and Kandel 1990). They may therefore work in concert as associative mechanisms to confer temporal specificity and other properties of conditioning. Other forms of plasticity also exist within the siphon sensory cells, such as increases in excitability (Klein et al. 1986; Walters 1987; Mercer et al. 1991), as well as at other loci in the siphon-withdrawal circuit (Colebrook and Lukowiak 1988; Frost et al. 1988; Wright et al. 1991; Trudeau and Castellucci 1992; Fischer and Carew 1993), and these may also play a role. Nonetheless, our present results indicate that the properties of activity-dependent enhancement of presynaptic facilitation in siphon sensory cells display striking parallels to several critical features of temporal specificity of classical conditioning and may thus contribute to their expression on a behavioral level.

\section{Acknowledgments}

This research was supported by Office of Naval Research grant N000014-89J-1954, a Pew Biomedical Scholars Award, and an Alfred P. Sloan Research Fellowship to G.A.C. and by National Institute of Mental Health grant 26212 to R.D.H. E.R.K. is supported by the Howard Hughes Medical Institute. We thank Drs. Thomas W. Abrams and David L. Glanzman for comments on a previous version of the manuscript.

The publication costs of this article were defrayed in part by payment of page charges. This article must therefore be hereby marked "advertisement" in accordance with 18 USC section 1734 solely to indicate this fact.

\section{References}

Abrams, T.W. 1985. Activity-dependent presynaptic facilitation: An associative mechanism in Aplysia. Cell. Mol. Neurobiol. 5: 123-145.

Alkon, D.L. 1979. Voltage-dependent calcium and potassium ion conductances: A contingency mechanism for an associative learning model. Science 205: 810-816.
1980. Membrane depolarization accumulates during acquisition of an associative behavioral change. Science 210: $1375-1376$

Baxter, D.A. and J.H. Byrne. 1989. Serotonergic modulation of two potassium currents in the pleural sensory neurons of Aplysia. J. Neurophysiol. 62: 665-679.

1990. Differential effects of cAMP and serotonin on membrane current, action potential duration, and excitability in somata of pleural sensory neurons of Aplysia. J. Neurophysiol. 64: 978-990.

Belardetti, F., S. Schacher, E.R. Kandel, and S. Siegelbaum. 1986. The growth cones of Aplysia sensory neurons: Modulation by serotonin of action potential duration and single potassium channel currents. Proc. Natl. Acad. Sci. 83: 7094-7098.

Braha, O., N. Dale, B. Hochner, M. Klein, T.W. Abrams, and E.R. Kandel. 1990. Second messengers involved in the two processes of presynaptic facilitation that contribute to sensitization and dishabituation in Aplysia sensory neurons. Proc. Natl. Acad. Sci. 87: 2040-2044.

Brunelli, M., V. Castellucci, and E.R. Kandel. 1976. Synaptic facilitation and behavioral sensitization in Aplysia: Possible role of serotonin and cyclic AMP. Science 194: 1178-1181.

Byrne, J.H., V.F. Castellucci, T.J. Carew, and E.R. Kandel. 1978. Stimulus-response relations and stability of mechanoreceptor and motor neurons mediating defensive gill-withdrawal reflex in Aplysia. I. Neurophysiol. 41: 402-417.

Byrne, J.H., R. Zwartjes, R. Homayouni, S.D. Critz, and A. Eskin. 1993. Roles of second messenger pathways in neuronal plasticity and in learning and memory. Insights gained from Aplysia. Adv. Second Messenger Phosphoprotein Res. 27: 47-108.

Carew, T.J., E.T. Walters, and E.R. Kandel. 1981. Classical conditioning in a simple withdrawal reflex in Aplysia californica. I. Neurosci. 1: 1426-1437.

Carew, T.J., R.D. Hawkins, and E.R. Kandel. 1983. Differential classical conditioning of a defensive withdrawal reflex in Aplysia californica. Science 219: 397-400.

Carew, T.J., R.D. Hawkins, T.W. Abrams, and E.R. Kandel. 1984. A test of Hebb's postulate at identified synapses which mediate classical conditioning in Aplysia. J. Neurosci.

4: 1217-1224.

Chen, C. and R.F. Thompson. 1992. Associative long-term depression revealed by field potential recordings in rat cerebellar slice. Soc. Neurosci. Abstr. 18: 1215.

Colebrook, E. and K. Lukowiak. 1988. Learning by the Aplysia model system: Lack of correlation between gill and gill motor neurone responses. I. Exp. Biol. 135: 411-429.

Connor, J.A. and C.F. Stevens. 1971. Prediction of repetitive

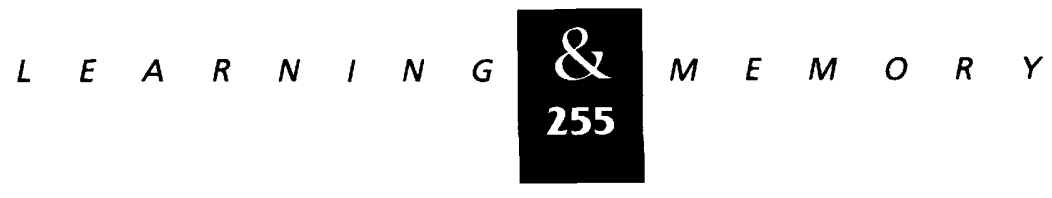




\section{Clark et al.}

firing behavior from voltage clamp data on an isolated neurone soma. J. Physiol. 213: 31-53.

Cui, M. and E.T. Walters. 1994. Homosynaptic LTP and PTP of sensorimotor synapses mediating the tail withdrawal reflex in Aplysia are reduced by postsynaptic hyperpolarization. Soc. Neurosci. Abstr. 20: 1071.

Dale, N., S. Schacher, and E.R. Kandel. 1988. Long-term facilitation in Aplysia involves increase in transmitter release. Science 239: 282-285.

Disterhoft, J.F., D.A. Coulter, and D.L. Alkon. 1986. Conditioning-specific membrane changes of rabbit hippocampal neurons measured in vitro. Proc. Natl. Acad. Sci. 83: 2733-2737.

Eichenbaum, H., T. Otto, and N.J. Cohen. 1992. The hippocampus-What does it do? Behav. Neural Biol. 57: 2-36.

Ekerot, C.-F. and M. Kano. 1989. Stimulation parameters influencing climbing fibre long-term depression of parallel fibre synapses. Neurosci. Res. 6: 264-268.

Eliot, L.S. 1991. "Presynaptic modulation of Aplysia sensory-to-motor neuron synapses in culture." Ph.D. dissertation, Columbia University, New York.

Eliot, L.S., E.R. Kandel, S.A. Siegelbaum, and H. Blumenfeld. 1993. Imaging terminals of Aplysia sensory neurons demonstrates role of enhanced $\mathrm{Ca}^{2+}$ influx in presynaptic facilitation. Nature 361: 634-637.

Eliot, L.S., R.D. Hawkins, E.R. Kandel, and S. Schacher. 1994. Pairing-specific, activity-dependent presynaptic facilitation at Aplysia sensory-motor neuron synapses in isolated cell culture. J. Neurosci. 14: 368-383.

Fischer, T.M. and T.J. Carew. 1993. Activity-dependent potentiation of recurrent inhibition: A mechanism for dynamic gain control in the siphon withdrawal reflex of Aplysia. J. Neurosci. 13: 1302-1314.

Frost, W.N., G.A. Clark, and E.R. Kandel. 1988. Parallel processing of short-term memory for sensitization in Aplysia. J. Neurobiol. 19: 297-334.

Ghirardi, M., O. Braha, B. Hochner, P.G. Montarolo, E.R. Kandel, and N. Dale. 1992. Roles of PKA and PKC in facilitation of evoked and spontaneous transmitter release at depressed and nondepressed synapses in Aplysia sensory neurons. Neuron 9: 479-489.

Glanzman, D.L. 1994. Postsynaptic regulation of the development and long-term plasticity of Aplysia sensorimotor synapses in cell culture. J. Neurobiol. 25: 666-693.

1995. The cellular basis of classical conditioning in Aplysia californica-lt's less simple than you think. Trends Neurosci. 18: 30-36.

Glanzman, D.L. and X.Y. Lin. 1994. The cellular basis of
CS-US pairing efficacy for classical conditioning of Aplysia's withdrawal reflex: Potential contribution of a Hebbian mechanism. Neth. J. Zool. 44 (in press).

Glanzman, D.L., S.L. Mackey, R.D. Hawkins, A.M. Dyke, P.E. Lloyd, and E.R. Kandel. 1989. Depletion of serotonin in the nervous system of Aplysia reduces the behavioral enhancement of gill withdrawal as well as the heterosynaptic facilitation produced by tail shock. I. Neurosci. 9: 4200-4213.

Goldsmith, B.A. and T.W. Abrams. 1991. Reversal of synaptic depression by serotonin at Aplysia sensory neuron synapses involves activation of adenylyl cyclase. Proc. Natl. Acad. Sci. 88: 9021-9025.

1992. cAMP modulates multiple $\mathrm{K}^{+}$currents, increasing spike duration and excitability in Aplysia sensory neurons. Proc. Natl. Acad. Sci. 89: 11481-11485.

Gustafsson, B. and H. Wigström. 1986. Hippocampal long-lasting potentiation produced by pairing single volleys and brief conditioning tetani evoked in separate afferents. $/$. Neurosci. 6: 1575-1582.

Hawkins, R.D. 1981. Interneurons involved in mediation and modulation of gill-withdrawal reflex in Aplysia. III. Identified facilitating neurons increase $\mathrm{Ca}^{2+}$ current in sensory neurons. I. Neurophysiol. 45: 327-339.

1989. Localization of potential serotonergic facilitator interneurons in Aplysia by glyoxylic acid histofluorescence combined with retrograde fluorescent labeling. J. Neurosci. 9: 4214-4226.

Hawkins, R.D. and T.W. Abrams. 1984. Evidence that activity-dependent facilitation underlying classical conditioning in Aplysia involves modulation of the same ionic current as normal presynaptic facilitation. Soc. Neurosci. Abstr. 10: 268.

Hawkins, R.D. and E.R. Kandel. 1990. Hippocampal LTP and synaptic plasticity in Aplysia: Possible relationship of associative cellular mechanisms. Semin. Neurosci. 2: $391-401$.

Hawkins, R.D. and S. Schacher. 1989. Identified facilitator neurons L29 and L28 are excited by cutaneous stimuli used in dishabituation, sensitization, and classical conditioning of Aplysia. I. Neurosci. 9: 4236-4245.

Hawkins, R.D., V.F. Castellucci, and E.R. Kandel. 1981 a. Interneurons involved in mediation and modulation of gill-withdrawal reflex in Aplysia. I. Identification and characterization. J. Neurophysiol. 45: 304-314.

1981b. Interneurons involved in mediation and modulation of gill-withdrawal reflex in Aplysia. II. Identified neurons produce heterosynaptic facilitation contributing to behavioral sensitization. J. Neurophysiol. 45: 315-326.

Hawkins, R.D., T.W. Abrams, T.J. Carew, and E.R. Kandel. 1983. A cellular mechanism of classical conditioning in 
Aplysia: Activity-dependent amplification of presynaptic facilitation. Science 219: 400-405.

Hawkins, R.D., T.J. Carew, and E.R. Kandel. 1986. Effects of interstimulus interval and contingency on classical conditioning of the Aplysia siphon withdrawal reflex. ). Neurosci. 6: 1695-1701.

Hawkins, R.D., N. Lalevic, G.A. Clark, and E.R. Kandel. 1989. Classical conditioning of the Aplysia siphon-withdrawal reflex exhibits response specificity. Proc. Natl. Acad. Sci. 86: 7620-7624.

Hearst, E. 1988. Fundamentals of learning and conditioning. In Stevens' handbook of experimental psychology, 2nd ed. Vol. 2. Learning and cognition (ed. R.C. Atkinson, R.). Herrnstein, G. Lindzey, and R.D. Luce), pp. 3-109. John Wiley \& Sons, New York.

Hochner, B. and E.R. Kandel. 1992. Modulation of a transient $\mathrm{K}^{+}$current in the pleural sensory neurons of Aplysia by serotonin and CAMP: Implications for spike broadening. Proc. Natl. Acad. Sci. 89: 11476-11480.

Hochner, B., M. Klein, S. Schacher, and E.R. Kandel. 1986a. Action potential duration and the modulation of transmitter release from the sensory neurons of Aplysia in presynaptic facilitation and behavioral sensitization. Proc. Natl. Acad. Sci. 83: 8410-8414.

1986b. Additional component in the cellular mechanism of presynaptic facilitation contributes to behavioral dishabituation in Aplysia. Proc. Natl. Acad. Sci. 83: 8794-8798.

Kandel, E.R. and J.H. Schwartz. 1982. Molecular biology of learning: Modulation of transmitter release. Science 218: $433-443$.

Kelso, S.R., A.H. Ganong, and T.H. Brown. 1986. Hebbian synapses in hippocampus. Proc. Natl. Acad. Sci. 83: $5326-5330$.

Klein, M. 1993. Differential cyclic AMP dependence of facilitation at Aplysia sensorimotor synapses as a function of prior stimulation: Augmentation versus restoration of transmitter release. I. Neurosci. 13: 3793-3801.

1994. Synaptic augmentation by $5-\mathrm{HT}$ at rested Aplysia sensorimotor synapses: Independence of action potential prolongation. Neuron 13: 159-166.

Klein, M. and E.R. Kandel. 1978. Presynaptic modulation of a voltage-dependent $\mathrm{Ca}^{2+}$ current: Mechanism for behavioral sensitization. Proc. Natl. Acad. Sci. 75: 3512-3516.

1980. Mechanism of calcium current modulation underlying presynaptic facilitation and behavioral sensitization in Aplysia. Proc. Natl. Acad. Sci. 77: 6912-6916.

Klein, M., J. Camardo, and E.R. Kandel. 1982. Serotonin modulates a specific potassium current in the sensory neurons that show presynaptic facilitation in Aplysia. Proc. Natl. Acad. Sci. 79: 5713-5717.

Klein, M., B. Hochner, and E.R. Kandel. 1986. Facilitatory transmitters and CAMP can modulate accommodation as well as transmitter release in Aplysia sensory neurons: Evidence for parallel processing in a single cell. Proc. Natl. Acad. Sci. 83: 7994-7998.

Lin, X.Y. and D.L. Glanzman. 1994a. Hebbian induction of long-term potentiation of Aplysia sensorimotor synapses: Partial requirement for activation of a NMDA-related receptor. Proc. R. Soc. Lond. B. 255: 215-221.

1994b. Long-term potentiation of Aplysia sensorimotor synapses in cell culture: Regulation by postsynaptic voltage. Proc. R. Soc. Lond. B. 255: 113-118.

Mackey, S.L., D.L. Glanzman, S.A. Small, A.M. Dyke, E.R. Kandel, and R.D. Hawkins. 1987. Tail shock produces inhibition as well as sensitization of the siphon-withdrawal response of Aplysia: Possible behavioral role for presynaptic inhibition mediated by the peptide Phe-Met-Arg-Phe-NH2. Proc. Natl. Acad. Sci. 84: 8730-8734.

Mackey, S.L., E.R. Kandel, and R.D. Hawkins. 1989. Identified serotonergic neurons LCB1 and RCB1 in the cerebral ganglia of Aplysia produce presynaptic facilitation of siphon sensory neurons. J. Neurosci. 9: 4227-4235.

Mercer, A.R., N.J. Emptage, and T.J. Carew. 1991. Pharmacological dissociation of modulatory effects of serotonin in Aplysia sensory neurons. Science 254: 1811-1813.

Moscovitch, A. and V.M. LoLordo. 1968. Role of safety in the Pavlovian backward fear conditioning procedure. /. Comp. Physiol. Psychol. 66: 673-678.

Occor, K.A., E.T. Walters, and J.H. Byrne. 1985. Associative conditioning analog selectively increases CAMP levels of tail sensory neurons in Aplysia. Proc. Natl. Acad. Sci. 82: 2548-2552.

Rescorla, R.A. 1988. Behavioral studies of Pavlovian conditioning. Annu. Rev. Neurosci. 11: 329-352.

Sacktor, T.C. and J.H. Schwartz. 1990. Sensitizing stimuli cause translocation of protein kinase $C$ in Aplysia sensory neurons. Proc. Natl. Acad. Sci. 87: 2036-2039.

Sejnowski, T.J., S. Chattarji, and P.K. Stanton. 1990. Homosynaptic long-term depression in hippocampus and neocortex. Semin. Neurosci. 2: 355-363.

Siegelbaum, S.A., J.S. Camardo, and E.R. Kandel. 1982. Serotonin and cyclic AMP close single $\mathrm{K}^{+}$channels in Aplysia sensory neurons. Nature 299: 413-417.

Squire, L.R. 1992. Memory and the hippocampus: A synthesis from findings with rats, monkeys and humans. Psychol. Rev. 99: 195-231.

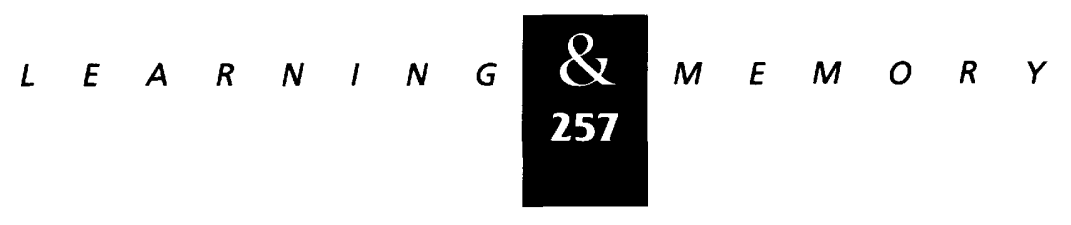




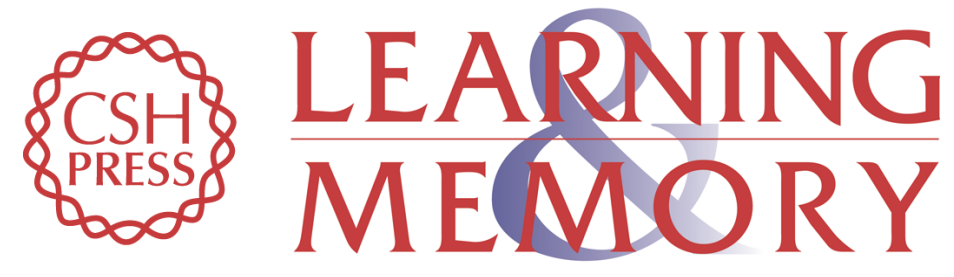

\section{Activity-dependent enhancement of presynaptic facilitation provides a cellular mechanism for the temporal specificity of classical conditioning in Aplysia.}

G A Clark, R D Hawkins and E R Kandel

Learn. Mem. 1994, 1:

Access the most recent version at doi:10.1101//m.1.4.243

References This article cites 75 articles, 42 of which can be accessed free at: http://learnmem.cshlp.org/content/1/4/243.full.html\#ref-list-1

License

Email Alerting Receive free email alerts when new articles cite this article - sign up in the box at the Service top right corner of the article or click here. 